\title{
Técnica de plicatura modificada de túnica albugínea para la corrección de la incurvación peneana
}

\author{
Pascual Regueiro D, Rodríguez Vela L, Gonzalvo Ibarra A. \\ Departamento de Urología. Hospital Universitario “Miguel Servet”. Zaragoza.
}

Actas Urol Esp 2006;30(8):784-790

\section{RESUMEN}

TÉCNICA DE PLICATURA MODIFICADA DE TÚNICA ALBUGÍNEA PARA LA CORRECCIÓN DE LA INCURVACIÓN PENEANA

Presentamos nuestra experiencia en el tratamiento de la incurvación peneana, tanto secundaria a la enfermedad de Peyronie como a la de causas congénitas, mediante una técnica modificada de plicatura de túnica albugínea, utilizando puntos invertidos de Prolene ${ }^{\circledR}$, recubiertos de puntos invertidos de Vicryl ${ }^{\circledR}$. Los primeros confieren seguridad a la plicatura y los segundos tienen la función de "enterrar" a los primeros evitando las molestias secundarias al material irreabsorbible.

Palabras clave: Plicatura de túnica albuginea. Enfermedad de Peyronie. Incurvación peneana.

\section{ABSTRACT}

\section{MODIFICATED PLICATION TECHNIQUE OF THE TUNICA ALBUGINEA} IN PENIS CURVATURE CORRECTION

We present our penile curvature treatment experience in Peyronie's disease and cogenital curvature, using a modificated plication technique of the tunica albuginea with Prolene ${ }^{\circledR}$ inverted sutures covered with Vicryl ${ }^{\circledR}$ ones. The first suture gives security to the plication and the second one hides the first dots avoiding the nonabsorbable sutures bother.

Keywords: Tunica albuginea plication. Peyronie’s disease. Penile curvatura.

$\mathrm{L}$ a incurvación peneana, ya sea de origen congénito o bien secundario a la enfermedad de Peyronie, es un problema que con cierta frecuencia nos vemos obligados a abordar en nuestra labor diaria en una consulta de urología.

Esta incurvación motiva en el paciente una serie de molestias que van desde el mero rechazo estético, hasta la dificultad o imposibilidad completa para la relación sexual, ya sea por el grado de incurvación como por el dolor asociado a la misma.

La corrección de estas incurvaciones ha ido evolucionando con la aparición de distintas técnicas quirúrgicas cuyo fin principal es actuar sobre la convexidad de la curvatura para corregir la concavidad de la misma, consiguiendo así un enderezamiento peneano. Todas estas técnicas asocian un acortamiento peneano más o menos manifiesto.

Las diversas técnicas han intentado en mayor o menor medida el intentar reducir este acortamiento. Además, las distintas modificaciones han buscado conseguir una corrección persistente, evitando el fallo de las suturas (más frecuente en las realizadas con material reabsorbible), e intentando disminuir las molestias provocadas por la persistencia de las plicaturas realizadas con materiales no reabsorbibles.

Presentamos aquí nuestra experiencia en 47 pacientes tratados mediante una técnica de plicatura simple, es decir, sin resección de túnica albuginea, en la que la modificación consiste en 
realizar plicaturas mediante puntos invertidos de material irreabsorbible (Prolene ${ }^{\circledR}$ ), que aseguren las mismas, recubiertas de dos puntos invertidos de material reabsorbible $\left(\right.$ Vicry ${ }^{\circledR}$ ), que entierren las primeras, evitando así las molestias motivadas por la persistencia de las suturas.

\section{MATERIAL Y MÉTODOS}

Se lleva a cabo un análisis retrospectivo de 47 varones intervenidos quirúrgicamente en nuestro Servicio entre 1999 y 2003. En todos ellos se realizó una técnica de plicatura de túnica albugínea modificada como sistema para la corrección de la incurvación peneana.

Todos los pacientes presentaban una desviación peneana. De ellos, 27 pacientes (57\%) mostraban una incurvación congénita (C) y 20 pacientes $(42,5 \%)$ una desviación secundaria a enfermedad de Peyronie (P).

La edad media global fue de 37,6 años (18-69), siendo la edad media en el momento de la cirugía de 56,5 en los P y de 23,6 años en los C.

El motivo de consulta principal fue la dificultad para mantener una relación sexual (26 pacientes), seguido del dolor (17), motivos estéticos (16) e imposibilidad completa para la penetración (4), presentando algunos pacientes asociación de varios síntomas.

La incurvación predominante de los C $(51,8 \%)$ fue ventral, y en $\mathrm{P}$ presentaban un $45 \%$ dorsal y otro $45 \%$ lateral. (Fig. 1)

En el grupo de los C, sólo dos pacientes aquejaban una disfunción eréctil asociada de carácter leve o moderada, que se correspondía fundamentalmente a la ansiedad motivada por la dificultaddolor durante las relaciones sexuales.

En el Grupo de los P, el 60\% de los pacientes no mostraba ninguna alteración de su función sexual, mientras que el resto presentaba una disfunción eréctil de carácter leve a moderado. En estos últimos se comprobó la eficacia de fármacos inhibidores de la fosfodiesterasa por vía oral en la mejoría de su disfunción eréctil previamente a la corrección de la incurvación.

Ningún paciente había recibido tratamientos quirúrgicos previos para la corrección de la incurvación. El 80\% de los pacientes de P llevaron tratamiento médico de su enfermedad hasta que se comprobó la estabilización de las placas.

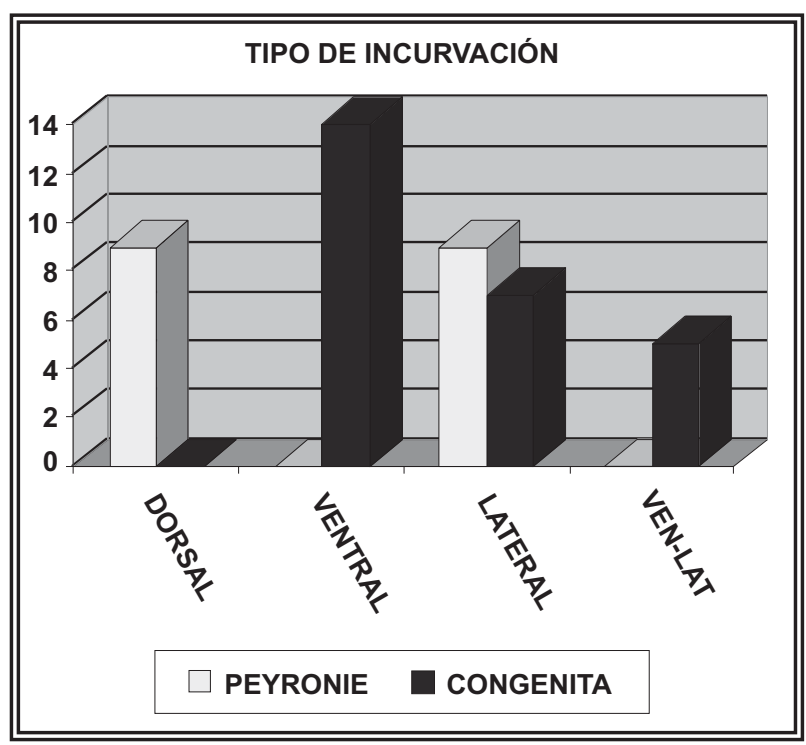

FIGURA 1. Distribución de los pacientes según el tipo de incurvación y el tipo de patología. En blanco podemos ver la distribución de los pacientes afectos de Peyronie y en negro los afectos de incurvación congénita.

Descripción de la técnica quirúrgica (Figs. 2 y 3)

La técnica empleada en el Servicio de Urología del Hospital Universitario "Miguel Servet" de Zaragoza, consiste en una modificación realizada sobre la técnica de plicatura simple, sin resección de parche de albuginea, que nos ha reportado excelentes resultados. La secuencia quirúrgica que practicamos es la siguiente:

1. Se realiza una incisión coronal, asociando la circuncisión reglada del prepucio si es redundante, seguida de la exposición de la fascia de Buck tras disección manual roma de la piel hasta la base peneana. Es importante liberar bien la piel para determinar de forma precisa el grado de incurvación.

2. Se practica entonces una erección artificial mediante la técnica de Gittes (inyección de suero salino templado, con banda compresiva en raíz del pene) para valoración del grado y dirección de la incurvación.

3. Se procede entonces a la disección longitudinal a nivel lateral de la fascia de Buck para alcanzar la túnica albugínea. La incurvación congénita de pene suele ser ventral, lo que implica la realización de plicaturas en la cara dorsal de ambos cuerpos cavernosos, por lo que se debe tener especial cuidado en evitar la lesión del haz neurovascular, que discurre en la región más 

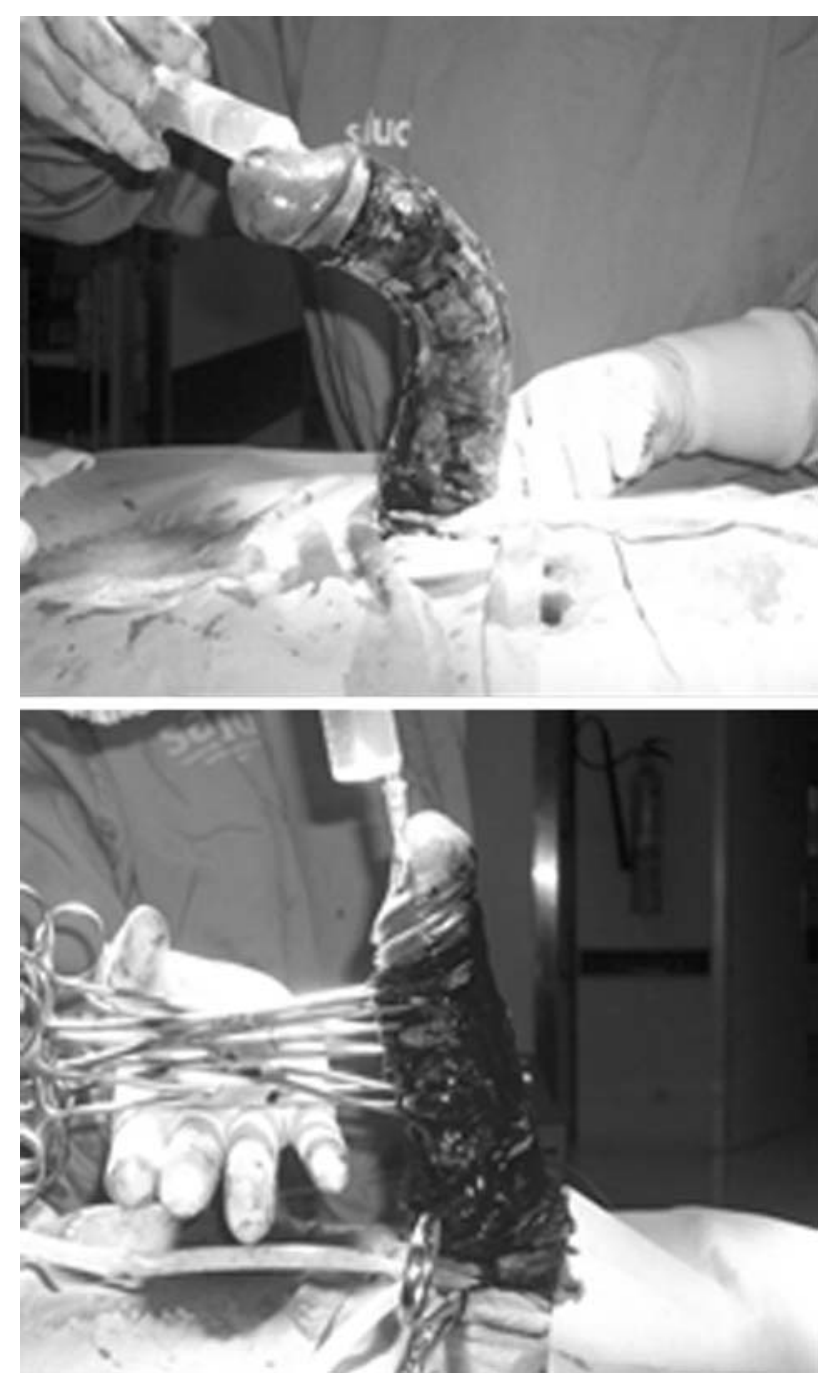

FIGURA 2. Técnica quirúrgica 1: A la derecha podemos ver el pene denudado hasta su base y la incurvación ventral pura. Erección artificial según técnica de Gittes. A la izquierda se aprecia la corrección de la incurvación mediante plicaturas realizadas con pinzas de Allis colocadas dorsalmente.

medial de la cara dorsal del pene, debajo de la fascia de Buck. Se debe practicar la disección abriendo la fascia de Buck lo más lateral posible y disecar hasta el haz neurovascular. Generalmente no es necesario levantar el paquete neurovascular y pueden realizarse las plicaturas en la región dorsal, muy pegadas a nervio y arteria dorsal pero con cuidado de no dañarlos.

4. Seguidamente, y con el pene en flacidez, se utilizan pinzas de Allis para realizar plicaturas de la túnica albugínea, en la parte opuesta a la zona de máxima curvatura. Se comprueba la corrección de la incurvación con técnica de Gittes, realizando las modificaciones oportunas hasta conseguir la corrección deseada.

5. En la zona marcada con las pinzas de Allis, y sin exéresis de la albugínea se realizan con bisturí frío dos incisiones paralelas en la misma, que no penetren en tejido eréctil.

6. A nivel de las incisiones de la túnica albugínea se realiza inicialmente un punto central invertido de material no reabsorbible (Prolene $^{\circledR}$ $3 / 0$ ), flanqueado por 2 puntos invertidos laterales de material reabsorbible (Vycril ${ }^{\circledR} 3 / 0$ ).

Se anuda primero el punto central de Prolene que queda totalmente enterrado al anudar los puntos laterales reabsorbibles. Esta técnica permite que no se produzcan ni se palpen abultamientos. Además, el punto no reabsorbible mantiene la plicatura y evita las reincurvaciones. Las plicaturas no deben ser mayores de $8 \mathrm{~mm}$ de anchura y deben realizarse las necesarias para corregir la incurvación.

7. Se comprueba mediante nueva erección artificial del enderezamiento peneano y se procede al cierre de la fascia de Buck y de la piel.

\section{RESULTADOS}

De todas las intervenciones, 38 de los procedimientos $(80,8 \%)$ se llevaron a cabo con anestesia general, y 9 con anestesia raquídea.

La vía de abordaje fue por circuncisión en todos los casos salvo dos, un $\mathrm{C}$ y un $\mathrm{P}$ en los que se emplearon incisiones laterales longitudinales.

El número de plicaturas realizadas (Fig. 4), varió entre 2 y 6 plicaturas, siendo preciso mayor número de plicaturas por término medio en los $\mathrm{C}$ que en los $\mathrm{P}$, con una media de 4,5 plicaturas en las desviaciones ventro-laterales, de 4,3 en las dorsales puras y ventrales puras, de 3,6 en las dorso-laterales y de 2,5 en las laterales puras, siendo claramente estas últimas las de más fácil corrección.

El tiempo de cirugia fue inferior a los 90 minutos en el $95 \%$ de los $\mathrm{P}$ y en el $75 \%$ de las incurvaciones congénitas. La mayor duración de la cirugía en este último grupo viene justificada por el mayor número de plicaturas empleadas.

La media de ingreso, incluyendo el día de la cirugia fue de 2,7 días. A partir del año 2000, el tiempo de hospitalización, basándonos en los buenos resultados y escasas complicaciones y 

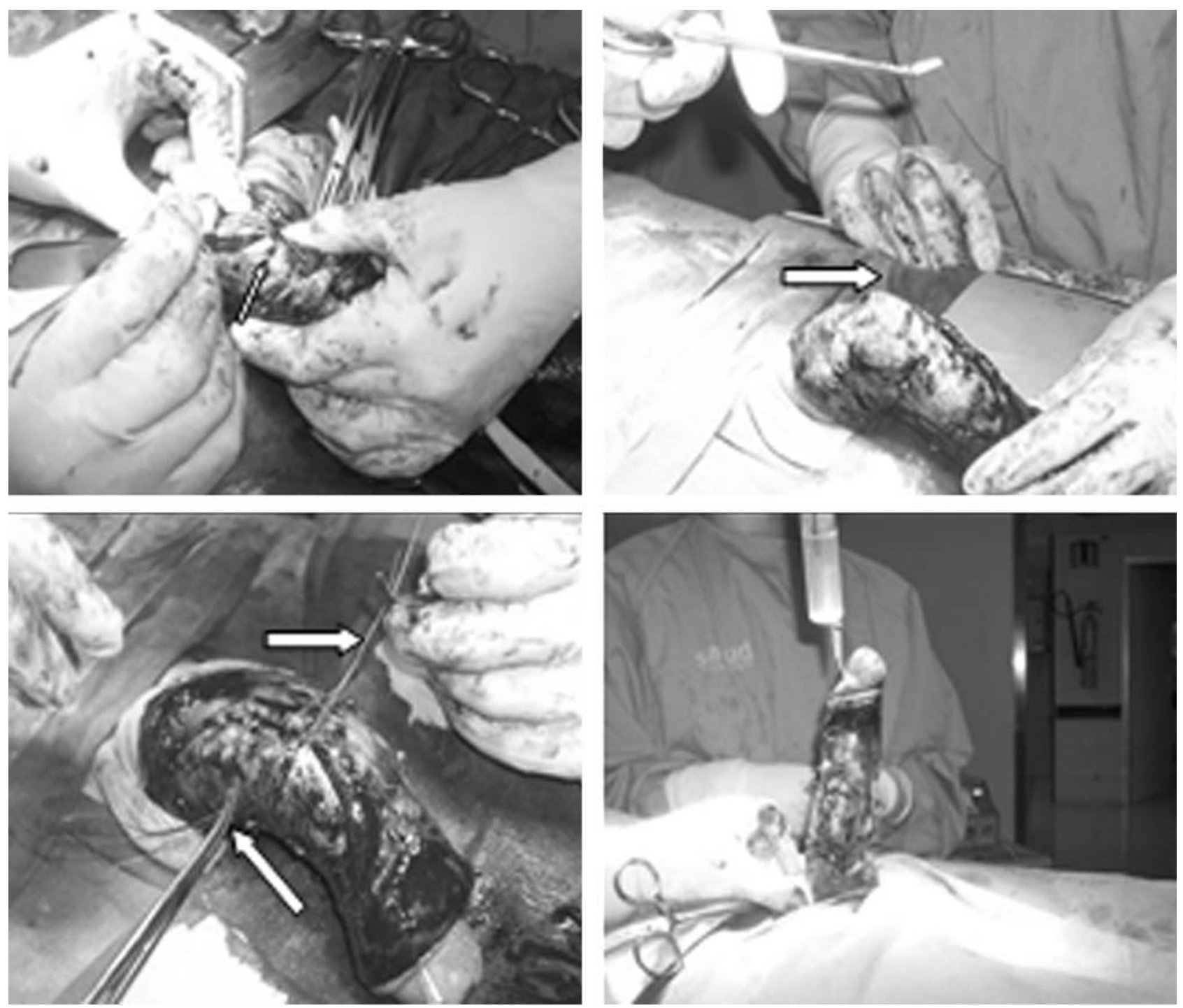

FIGURA 3. Técnica quirúrgica 2: Arriba a la izquierda, una vez disecada lateralmente la fascia de Buck, se realizan dos minimas incisiones con bisturi frio (flecha blanca) sobre las marcas de la plicatura realizada con las pinzas de Allis. Arriba a la derecha se aprecia el punto invertido con material irreabsorbible sobre las marcas del bisturi (flecha blanca), que va a constituir la plicatura. Abajo a la izquierda podemos ver el punto de plicatura anudado, flanqueado por dos puntos invertidos de material irreabsorbible (flechas blancas). Abajo a la derecha, nueva erección artificial donde se aprecia el resultado final tras la realización de todas las plicaturas.

molestias postoperatorias, se redujo en la mayor parte de los casos al día de la intervención, siendo dados de alta a la mañana siguiente de la misma.

Complicaciones inmediatas de la cirugía: dos reintervenciones por sangrado (uno de ellos por hidrocele intervenido conjuntamente) y dos hematomas que no requirieron ninguna actuación.

A la hora de valorar el resultado quirúrgico, se observó una corrección completa en el 75\% de los
$\mathrm{P}$ y en el 96,3\% de los $\mathrm{C}$, mientras que en 5 pacientes de $\mathrm{P}$ y 1 de $\mathrm{C}$ se consiguió una corrección parcial pero suficiente para mantener una relación sexual normal y sin molestias.

El 92,6\% de los C refirieron una mejoría de la actividad sexual, los 2 pacientes restantes no mejoraron por la aparición progresiva de disfunción eréctil. En el caso de los $\mathrm{P}$ el 80\% mejoraron en sus relaciones sexuales mientras que 4 pacientes no mejoraron por progresión de su disfunción eréctil. 


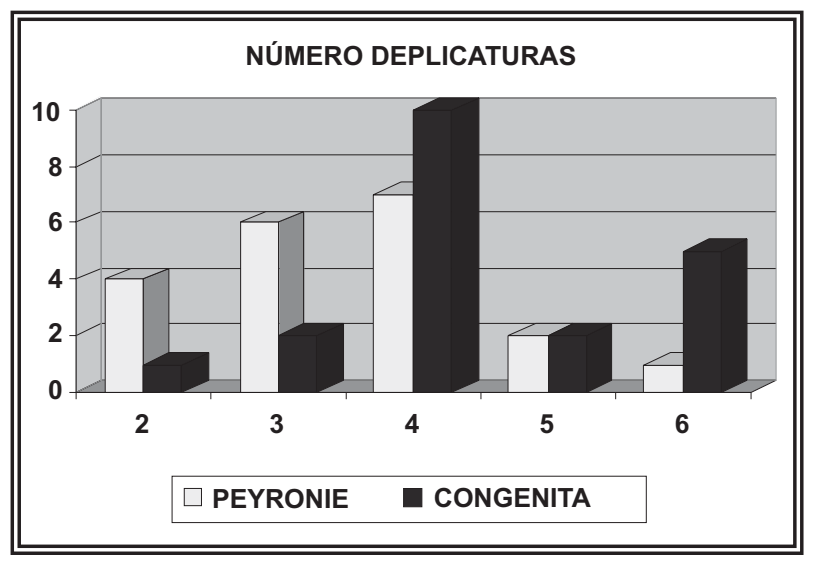

FIGURA 4. Distribución de los pacientes según el número de plicaturas necesarias para corregir la incurvación y el tipo de patología. En blanco podemos ver la distribución de los pacientes afectos de Peyronie y en negro los afectos de incurvación congénita.

A la hora de la valoración subjetiva por parte del paciente, se les invitó a que clasificaran como muy buenos, buenos o malos los resultados estéticos y funcionales de la intervención. De esta manera el 79\% valoró la intervención y sus resultados como muy buenos, el $19 \%$ como buenos y únicamente un paciente valoró los resultados como malos. Este último necesitó de reintervención por sangrado en el postoperatorio inmediato, y presentó una progresión rápida de su disfunción eréctil con mala respuesta a los fármacos orales.

En la valoración por grupos, el $100 \%$ de los C y el $85 \%$ de los $\mathrm{P}$ se encontraban contentos con los resultados.

El seguimiento postoperatorio se realizó en nuestras consultas de andrología. A los seis meses de la cirugía todos los pacientes se encontraban dados de alta de su patología, con una media de dos consultas postoperatorias.

Durante este seguimiento se pudieron observar que el $77 \%$ de los pacientes del grupo C no presentó ninguna complicación, al igual que el $55 \%$ del grupo de los P. En ambos grupos hubo un caso de molestias provocadas por los puntos de sutura que obligaron a reintervenir a uno de ellos, perteneciente al grupo $\mathrm{C}$, observándose un mal enterramiento de las suturas.

Dos pacientes se quejaron de acortamiento significativo de la longitud peneana, ambos correspondian a incurvaciones complejas que requirieron seis plicaturas.
Un paciente sufrió dolores posquirúrgicos que se aliviaron con la toma de antiinflamatorios.

Dos pacientes aquejaron disminución de sensibilidad en el glande que se recuperó a los tres meses de la cirugía.

En el grupo de los P, lógicamente al tratarse de un grupo de mayor edad, se observó la aparición o agudización de su disfunción eréctil en 8 pacientes, en dos de los cuales fue debido a progresión de su enfermedad de Peyronie. El resto mejoró con tratamiento oral.

En dos pacientes del grupo $\mathrm{P}$ y en uno del grupo $\mathrm{C}$ se observó reincurvación tras la cirugía $(6,3 \%)$, uno de los cuales fue debida a progresión de su enfermedad. En dos de ellos esta reincurvación no les dificultaba la penetración, pudiendo mantener una relación sexual normal.

\section{DISCUSIÓN}

Nesbit, en 1965 sentó las bases de las modernas técnicas de plicatura, al practicar en tres pacientes con incurvación congénita del pene una intervención consistente en acortar el lado convexo del pene mediante la resección de elipses de la túnica albuginea ${ }^{1}$. Pryor et al., en 1979 proponen la utilización de la técnica de Nesbit en la enfermedad de Peyronie $^{2}$ y en 1995 publican su experiencia de 16 años con 359 pacientes obteniendo un resultado global excelente ${ }^{3}$. El defecto que se crea en túnica albugínea se cierra con sutura no reabsorbible, con los nudos invertidos, orientados hacia el interior del cuerpo cavernoso, con el fin de que no sean molestos en el momento de la erección o el coito. Las suturas reabsorbibles son causa frecuente de fracaso.

Sassine $^{4}$ y Licht ${ }^{5}$ realizan la plicatura mediante una incisión longitudinal en la túnica albugínea con cierre horizontal de la misma, utilizando material no reabsorbible y sutura continua. Daitch ${ }^{6}$ describe unos resultados excelentes a largo plazo con esta variante de corporoplastia. La gran complicación de esta técnica es que la sutura crea abultamientos "orejones" que se palpan, se ven y son muy mal tolerados por los pacientes.

Rehman crea un lecho cruento que facilite la cicatrización y permanencia de la plicatura, bien mediante una pequeña incisión de la túnica o bien mediante una escarificación o "afeitado" de la misma entre los puntos de plicatura? ${ }^{7}$. 
La plicatura simple ${ }^{8}$ supone la simplificación máxima de la técnica original de Nesbit pues prescinde de cualquier incisión y propone simplemente plicaturas múltiples de la túnica albugínea en la zona de máxima convexidad del pene. Fue popularizada por Essed y Schroeder $^{9,10}$ y Knispel $^{11}$. Además del inevitable acortamiento, la complicación más frecuente de la plicatura simple es que suele producir abultamientos a nivel de las plicaturas que se palpan y son motivo de queja por parte del paciente. La zona plicada no resecada produce una protusión y los puntos de sutura irreabsorbible a pesar de estar anudados de forma invaginante pueden llegar a molestar durante la erección o el coito.

En nuestro país, en 1993, Ruiz Castañé ${ }^{12}$, propuso realizar dos incisiones en la túnica albugínea sin resecar la elipse, a continuación, practica la plicatura hundiendo la albugínea en el interior del cuerpo cavernoso, minimizando las complicaciones de la técnica de Nesbit y evitando la exéresis de albugínea.

En las incurvaciones secundarias a la enfermedad de Peyronie, se han desarrollado técnicas quirúrgicas para la corrección de la incurvación y disminución de las molestias provocadas por la placa que llevan a cabo una exéresis completa de la misma. El defecto creado en la albuginea se ha corregido mediante la colocación de parches de muy diferente categoría. Así, Gelbard en 1989 describió el relleno de las soluciones de continuidad con injertos de fascia temporal, lo que sustituía un tejido rígido por uno elástico obteniendo buenos resultados. Das y Amar ${ }^{13}$ escogieron la túnica vaginal como material para los parches por la facilidad de obtención para el urólogo, sin embargo en grandes defectos no se obtenían buenos resultados.

Lockhart en 1991 obtuvo buenos resultados sustituyendo los parches por colgajos cremasterianos, que mejoraban así la vascularización del defecto de la albuginea ${ }^{14}$.

Lue y El-Sakka en 1998, basándose en la interpretación del cuerpo cavernoso como un gran vaso sanguíneo, obtuvieron buenos resultados con parches de vena safena ${ }^{15}$.

Hellstrom en $1994^{16,17}$ describió el uso de varios materiales heterólogos para la reparación del defecto creado tras la excisión de la placa, tales como pericardio cadavérico o incluso materiales inertes como el Silastic ${ }^{\circledR}$, Dacron ${ }^{\circledR}$, Gore$\mathrm{Tex}^{\circledR}$, con distintos resultados.

La técnica adoptada por nosotros, resulta adecuada para el tratamiento de las incurvaciones tanto debidas a la enfermedad de Peyronie como a las congénitas. Se trata de una intervención de sencilla realización, ya que no realizamos resección de albuginea $^{18}$ ni de las placas de Peyronie, por lo que no es necesaria la obtención de parches ni de colgajos para la corrección del defecto, que complican y alargan la cirugía. Como hemos podido ver, los resultados inmediatos y a largo plazo son buenos sin apenas complicaciones ${ }^{19}$, con una corrección funcional y estética adecuada. La utilización de material irreabsorbible para realizar las plicaturas, sobre un mínimo lecho cruento, consistente en una pequeña escarificación de la albuginea, aseguran la perdurabilidad de las mismas evitándose la reincurvación. El enterramiento de las plicaturas mediante puntos invertidos de material reabsorbible proporciona un mayor confort, minimizando las molestias motivadas por el material de sutura.

\section{CONCLUSIONES}

La técnica modificada de plicatura de túnica albugínea es una técnica sencilla y fácilmente reproducible para el tratamiento de la incurvación peneana, ya sea congénita o secundaria a la enfermedad de Peyronie.

La mínima incidencia de complicaciones, tanto inmediatas como a medio-largo plazo, el reducido tiempo quirúrgico, así como el de ingreso y la prácticamente nula reaparición de la reincurvación en los casos estudiados, hacen de esta técnica quirúrgica una cirugía electiva en nuestro medio para el tratamiento de este tipo de patología.

La utilización en otras series de material reabsorbible para la plicatura, favorecía el fallo de la misma y la reincurvación, por la debilitación temprana de la misma. Por otra parte, la utilización de material no reabsorbible provocaba en muchas ocasiones molestias en el paciente por la persistencia de la sutura fácilmente palpable bajo la piel. En nuestra técnica la incidencia de este tipo de complicaciones es mínima ya que la plicatura queda garantizada mediante una sutura irreabsorbible que por otra parte queda enterrada por otra reabsorbible que desaparece a los pocos días. 


\section{REFERENCIAS}

1. Nesbit RM. Congenital curvature of the phallus: report of three cases with description of corrective operations. J Urol. 1965;93:230-233.

2. Pryor JP, Fiztpatrick JM. A new approach to the correction of the penile deformity in Peyronie's disease. J Urol. 1979; 122(5):622-623.

3. Ralph DJ, Al Akraa M, Pryor JP. The Nesbit operation for Peyronie's disease: 16 year experience. J Urol. 1995;154 (4): 1362-1363.

4. Sassine AM, Wespes E, Sculman CC. Modified corporoplasty for penile curvature: 10 years experience. Urology 1994;44(3):419-421.

5. Licht MR, Lewis RW. Modified Nesbit procedure for the treatment of Peyronie's disease: a comparative outcome analysis. J Urol. 1997;158(2):460-463.

6. Daitch JA, Angermeier KW, Montague DK. Modified corporoplasty for penile curvature: Long term results and patient satisfaction. J Urol. 1999;162(6):2006-2009.

7. Rehman J, Benet A, Minsky LS, Melman A. Results of surgical treatment for abnormal penile curvature: Peyronie's disease and congenital deviation by modified Nesbit plication (tunical shaving and plication). J. Urol. 157(4):12881291.

8. Gholami SS, Lue TF. Correction of penile curvatura using the 16-dot plication technique: a review of 132 patients. J Urol. 2002 May; 167(5):2066-2069.

9. Essed E, Schroeder FH. New surgical treatment for Peyronie`s disease. Urology 1985;25(6):582-587.

10. Bosas P, Bosas L. Matjosaitis. Penis deviation correction using Schroder's method an patients posoperative quality of life. Medicina (Kaunas) 2002;38 (Suppl 1):63-65.

11. Knispel HH, Gonnerman D y Huland H: Modified surgical technique to correct congenital and acquired penile curvature. Eur Urol 1991;20(2):107-112.
12. Ruiz Castañé E, Segura A, Sarquella J. Corporoplastia como tratamiento conservador en la incurvación peneana. Resultados. Libro de Ponencias y Comunicaciones del VI Congreso Nacional de Andrología. Vigo (Pontevedra) 1993.

13. Dad S, Amar AD. Peyronie's disease: excision of the plaque and grafting with tunica vaginalis. Urol Clin North Am. 1982 Feb;9(1):197-201.

14. Lockhart JL. Personal comunication. Department of Urology, University of Southern Florida, Tampa 1991.

15. Lue TF, El-Sakka AI. Venous match grafo for Peyronie's disease: I. Technique. J Urol 1998;160(6 Pt 1):2047-2049.

16. Hellstronm WJ, Bivalacqua TJ. Peyronie's disease: etiology, medical and surgical therapy. J Androl. 2000 MayJun;21(3):347-354.

17. Hellstrom WJG, Reddy S. Application of pericardial graft in the surgical managemant of Peyronie's disease. J Urol. 2000;163(5): 1445-1447.

18. Schneider T, Sperling H, Schenck M, Schneider U, Rubben H. Treatment of penile curvatura-how to combine the adventages of simple plication and the Nesbit-procedure bysuperficial excision of the tunica albuginea. World $\mathrm{J}$ Urol. 2003;20(6):350-355.

19. Syed AH, Abbasi Z, Hargreave TB. Nesbit procedure for disabling Peyronie's curvatura: a median follow-up of 84 months. Urology 2003;61(5):999-1003.

\section{Dr. D. Pascual Regueiro}

E-mail: dpregueiro@mixmail.com

(Trabajo recibido el 3 de mayo de 2006) 